Article

\title{
Electrically Conductive Polyacrylamide-Polyaniline Superabsorbing Polymer Hydrogels
}

\author{
Nedal Abu-Thabit ${ }^{1, *}$ and Yunusa Umar ${ }^{2}$ \\ ${ }^{1}$ Department of Chemical and Process Engineering Technology, Jubail Industrial College, Jubail \\ Industrial City 31961, Saudi Arabia; E-Mail: abuthabit_nidal@yahoo.com \\ 2 Department of Chemical and Process Engineering Technology, Jubail Industrial College, Jubail \\ Industrial City 31961, Saudi Arabia; E-Mail: Umar_y@jic.edu.sa \\ * Author to whom correspondence should be addressed; E-Mail abuthabit_nidal@yahoo.com \\ Tel.: +966-13-340 2356
}

Received: 30 March 2014 / Accepted: 15 May 2014 / Published: 26 May 2014

\begin{abstract}
:
This paper reports two-steps methodology for preparation of electrically conductive superabsorbing polyacrylamide/ polyaniline hydrogel. In the first step, polyacrylamide superabsorbing hydrogels were prepared with high water swelling capacity $(\approx 500 \mathrm{~g}$ water/g polyacrylamide). In the second step, polyaniline was penetrated through the preformed polyacrylamide hydrogel networks with simple oxidative polymerization procedure. Depending on the synthesis conditions, polyacrylamide hydrogels were either fully or partially penetrated. The prepared conductive hydrogels exhibited electrical resistance in the range of 100-1000 Ohm/Sq., according to the employed doping electrolyte. The conductive hydrogels are proposed as potential conductive flexible electrodes for supercapacitor applications.
\end{abstract}

Keywords: hydrogel, conductive hydrogel, polyaniline, polyacrylamide, superabsorbing polymer. 


\section{Introduction}

Hydrogels are three dimensional networks that are chemically or physically crosslinked with excellent water swelling capacity. Electrically conductive hydrogels (ECHs) are special class of hydrogels with potential applications in areas such as bioactive electrode coating [1], actuators [2] and tissue engineering [3]. Electrically conductive hydrogels can be prepared by different methods such as, adding conductive metallic nanoparticles to the hydrogels [4, 5], use of electrically conductive polymer nanoparticles [6] and preparation of composite hydrogels by incorporation of electrically conductive polymers into the network structure of the hydrogel [7].

Electrically Conductive Polymers (ECPs) belong to those classes of polymers which have $\pi$ conjugation along the polymer backbone such as polyaniline (PANi), polypyrole (PPy) and polythiophene (PTh). Recently, electrically conductive polymer hydrogels (ECPHs) have been investigated for number of electrochemical devices applications [8]. ECPHs possess number of advantages such as providing improved electrode interface between the electronic and ionic transport phases, possibility of casting into different, complex and flexible shapes electrodes, and the possibility for preparation of micropatterns by ink-jet printing or spray coating.

Polyacrylamide (PAAM) hydrogels are one of the most studied superabsorbing polymers for applications such as drilling fluids [9], agriculture [10] and tissue engineering [11]. Non-conductive PAAM hydrogels can be converted into ECPHs by in situ polymerization of the ECPs into the preformed PAAM hydrogel network. Lira et al. employed electrochemical polymerization methodology for preparation of semi-interpenetrating networks of PANi/PAAM ECPHs and tested their possible applications as electrochemically controlled drug delivery device [12]. In this case, the electrically conductive polyaniline is triggered by electric pulse that will result in a drug release response. Tang et al. prepared superabsorbing poly(acrylate-aniline) and poly(acrylamide-aniline) conducting hydrogels by in situ polymerization procedure $[13,14]$. The first step includes preparation of crosslinked polyacrylamide hydrogel in the powder form using NMBA as a crosslinker, followed by in situ polymerization of absorbed aniline inside the swollen hydrogel powder by employing potassium persulfate as redox initiator.

In the current study, we report the synthesis of polyacrylamide/polyaniline conducting hydrogel by using a two-step procedure (figure 1). In the first step, PAAM chains were crosslinked by using formaldehyde (FA) as a crosslinker and then polyaniline was polymerized in different doping acidic media, which are, $\mathrm{HCl}, \mathrm{H}_{2} \mathrm{SO}_{4}$ and $\mathrm{H}_{3} \mathrm{PO}_{4}$. The synthesis conditions for the first step were optimized to obtain the PAAM hydrogels with the best mechanical properties.

\section{Results and Discussion}

\subsection{Preparation of polyacrylamide hydrogels}

In the current study, PAAM hydrogels were prepared by using formaldehyde crosslinker, as a replacement for the conventional NMBA crosslinker. In the first step, the addition of $\mathrm{HCl}$ as acid catalyst to adjust the $\mathrm{pH}$ was essential for formation of PAAM hydrogels $(\mathrm{pH} \approx 1)$, table 1 (entries 3- 
5). At neutral $\mathrm{pH}$, only soluble PAAM polymers were obtained, table 1 (entries 1 and 2). Hence, the proposed mechanism for such reaction is depicted in figure 1.

Table 1 Synthesis condition for PAAM hydrogels using FA as crosslinker and $\mathrm{HCl}$ as catalyst

\begin{tabular}{|c|c|c|c|c|c|l|}
\hline $\begin{array}{c}\text { Entry } \\
\text { No. }\end{array}$ & $\begin{array}{c}\text { Mass of } \\
\text { AAM } \\
\text { Monomer } \\
\mathbf{( g )}\end{array}$ & $\begin{array}{c}\text { Volume of } \\
\text { Water } \\
\mathbf{( m L )}\end{array}$ & $\begin{array}{c}\text { Volume } \\
\text { of 1M } \\
\mathbf{H C l} \\
\mathbf{( m L )}\end{array}$ & $\begin{array}{c}\text { Mass of } \\
\text { KPS } \\
\text { Initiator } \\
\mathbf{( g )}\end{array}$ & $\begin{array}{c}\text { Volume } \\
\text { of FA } \\
\text { Cross- } \\
\text { Linker } \\
\mathbf{( m L )}\end{array}$ & \multicolumn{1}{|c|}{$\begin{array}{c}\text { Physical Appearance of } \\
\text { PAAM Hydrogel }\end{array}$} \\
\hline 1 & 9.0 & 2 & 0 & 9.9 .0 & 1 & No gel, soluble polymer \\
\hline 2 & 9.0 & 9 & 0 & 9.9 .0 & 3 & No gel, soluble polymer \\
\hline 3 & 9.0 & 1 & 1 & 9.9 .0 & 1 & Transparent and brittle \\
\hline 4 & 0.9 & 2 & 1 & 0.015 & 0.10 & Transparent and slightly tough \\
\hline 5 & 9.0 & 2 & 1 & 9.9 .0 & 9.90 & $\begin{array}{l}\text { Transparent and slightly tough, \% } \\
\text { swelling = 510 }\end{array}$ \\
\hline
\end{tabular}

Figure 1 Proposed crosslinking mechanism of formaldehyde in acidic medium

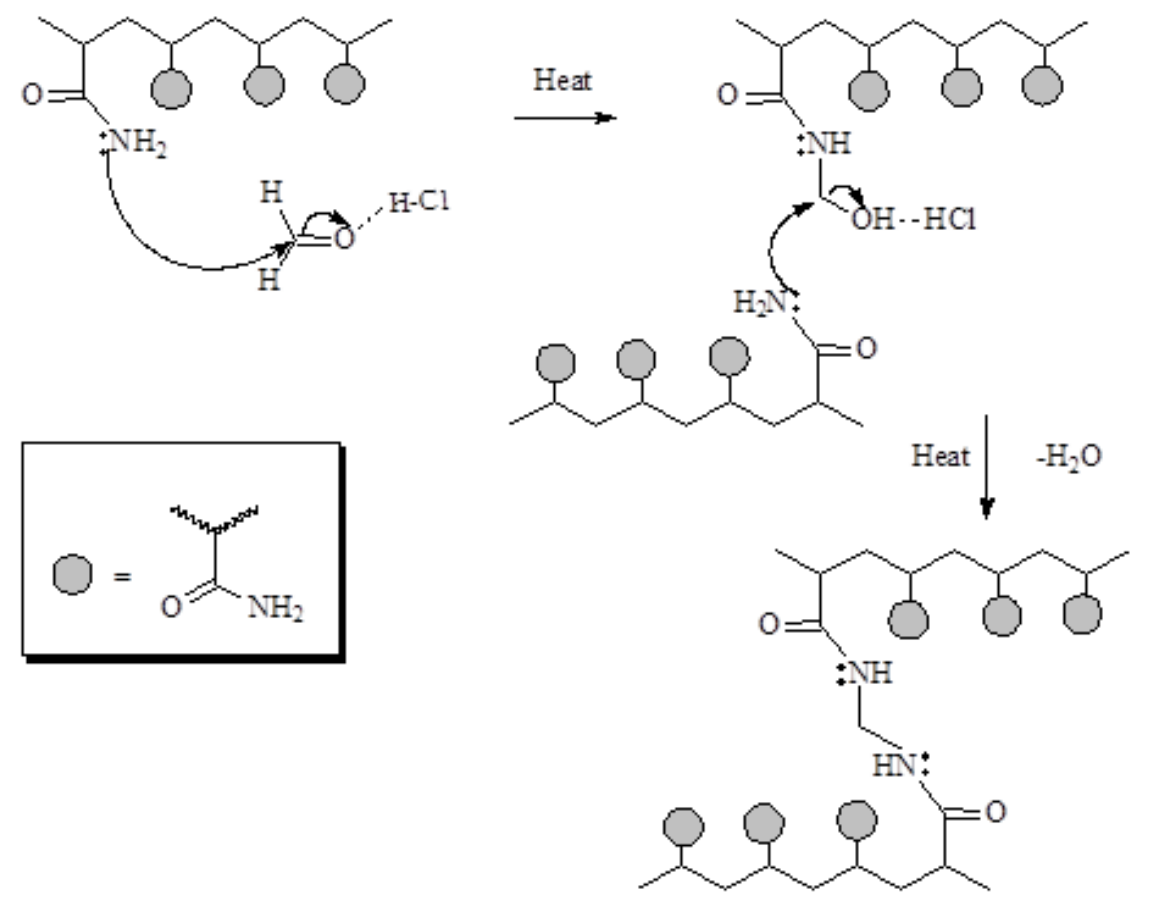

2.2 Preparation of Polyaniline/polyacrylamide hydrogels

Polyaniline was polymerized by oxidative polymerization. In the first step, aniline monomer was absorbed into PAAM hydrogels with certain doping acid. Then, polymerization was initiated by KPS as redox initiator. The adsorption and polymerization times were found to have important effect on the final shape of the obtained PANI/PAAM hydrogels. As can be inferred from figure 2, insufficient adsorption and polymerization periods produced hydrogels with incomplete penetration of polyaniline (figure 2, b); while longer adsorption and polymerization periods enable complete interpenetration of polyaniline chains into PAAM chains (figure 2, c). 
Figure 2 Pictures of synthesized PAAM and PANI/PAAM hydrogels with different conditions.

a) Transparent PAAM hydrogel (up) and Dark green $\begin{aligned} & \text { b) PANI/PAAM-HCl hydrogel, with about 2mm } \\ & \text { penetration of PANI. The soaking time for the PAAM } \\ & \text { hydrogel in aniline-HCl solution was } 1 \text { hour and the } \\ & \text { polymerization time was } 2 \text { hours. }\end{aligned}$
c) PANI/PAAM-HCl hydrogel (bottom)
solution was 3 hours and the polymerization time was 12 hours.

As shown in table 2, depending on the type of employed acid dopant, the electrical resistance of the fully swollen hydrogels was found to be in the range of $100-1000 \mathrm{Ohm} / \mathrm{Sq}$. These preliminary results can be used for future investigations of such conductive hydrogels to be used as flexible electrodes for supercapacitor applications.

Table 2 List of the prepared PAAM and PANI/PAAM hydrogels doped with different acid.

\begin{tabular}{|l|c|c|c|}
\hline \multicolumn{1}{|c|}{ Hydrogel } & Doping Acid & $\begin{array}{c}\text { Water Swelling } \\
\text { (g water/g polymer) }\end{array}$ & $\begin{array}{c}\text { Electrical Resistance } \\
\text { (Ohm/Sq.) }\end{array}$ \\
\hline PAAM & - & 510 & - \\
\hline PANI/PAAM-HCl & $\mathrm{HCl}$ & 450 & 110 \\
\hline PANI/PAAM- $\mathrm{S}_{2} \mathrm{SO}_{4}$ & $\mathrm{H}_{2} \mathrm{SO}_{4}$ & 442 & 340 \\
\hline PANI/PAAM-H $\mathrm{PO}_{4}$ & $\mathrm{H}_{3} \mathrm{PO}_{4}$ & 400 & 970 \\
\hline
\end{tabular}

\section{Experimental}

\subsection{Synthesis of polyacrylamide hydrogels (PAAM-HG)}

The required mass of acrylamide monomer was weighed in cylindrical polypropylene plastic test tube. Then, the required volumes of $1 \mathrm{M} \mathrm{HCl}$ solution and distilled water were added to the test tube and the acrylamide monomer was dissolved. This was followed by the addition of formaldehyde crosslinker to the plastics test tube. Finally, the required amount of potassium persulfate (KPS) initiator was weighed and added to the test tube. The solution was mixed and homogenized for few minutes and then the closed plastic test tube was placed in oven at $80{ }^{\circ} \mathrm{C}$ for 3-4 hours to allow for complete gelation. The obtained cylindrical gels were soaked in deionized water for 3-4 days, with subsequent water change every day, to 
allow for removal of unreacted species. The transparent hydrogels were stored in DI water for subsequent use.

\subsection{Synthesis of polyaniline/polyacrylamide conductive hydrogels (PANI/PAAM-HG)}

To a $250 \mathrm{~mL}$ beaker equipped with magnetic stirrer, $100 \mathrm{~mL}$ of the $1 \mathrm{M}$ doping acid and $0.50 \mathrm{~g}$ aniline monomer were added. Water-swollen PAAM-HGs were soaked in the prepared solution for certain period of time ( $2-12$ hours) to allow for penetration of anilinium chloride molecules into the PAAM network. After that, the required amount of KPS initiator was added to initiate polymerization of polyaniline. As the polymerization proceeded, the color of the solution and the PAAM hydrogel changed into dark green. After 12 hours reaction, the resulted hydrogels were removed and washed thoroughly with the corresponding doping acid solution. The obtained PANI/PAAM-HGs were further soaked in DI water for 3 days to allow for the removal of water soluble polyaniline oligomers. The obtained conductive hydrogels were stored in DI water for subsequent use.

\subsection{Swelling ratio measurements}

Swelling was measured by calculation of weight difference between swollen hydrogels in DI water (3 days) and dry weight of the perspective hydrogels (dried at $50^{\circ} \mathrm{C}$ ).

\subsection{Electrical resistance measurements}

Electrical resistance measurements were made according to the AATCC test method 76-2000, using Extech True-RMS digital multi-meter having a range from 0.1 to 4 x $10^{7} \mathrm{ohms}$.

\section{Conclusions}

The current study reports preparation of PAAM hydrogels by in situ crosslinking methodology utilizing formaldehyde as crosslinker in acidic media. The polymerization parameters were optimized to produce the best quality PAAM hydrogels. To obtain conductive hydrogels, polyaniline was polymerized inside the PAAM networks. To obtain fully penetrated polyaniline inside the PAAM networks, it was important to allow for sufficient absorption and polymerization periods of aniline monomers. The synthesized PANI/PAAM hydrogels were doped with different acids and exhibited a lowest electrical resistance $(110 \mathrm{Ohm} / \mathrm{Sq}$.) in case of using $\mathrm{HCl}$ as the doping acid. These preliminary results can be used for future investigations of such conductive hydrogels to be used as flexible electrodes for supercapacitor applications. Future work shall be devoted to improve the toughness and electrical conductivity of PANI/PAAM hydrogels.

\section{Conflicts of Interest}

The authors declare no conflict of interest. 


\section{References and Notes}

1. Gomez, N.; Schmidt, C. E., Nerve growth factor-immobilized polypyrrole: Bioactive electrically conducting polymer for enhanced neurite extension. Journal of Biomedical Materials Research Part A 2007, 81, (1), 135-149.

2. Guiseppi-Elie, A., Electroconductive hydrogels: synthesis, characterization and biomedical applications. Biomaterials 2010, 31, (10), 2701-2716.

3. Lee, K. Y.; Mooney, D. J., Hydrogels for tissue engineering. Chemical reviews 2001, 101, (7), 1869-1880.

4. Xiang, Y.; Chen, D., Preparation of a novel pH-responsive silver nanoparticle/poly (HEMAPEGMA-MAA) composite hydrogel. European Polymer Journal 2007, 43, (10), 4178-4187.

5. Devaki, S. J.; Narayanan, R. K.; Sarojam, S., Electrically conducting silver nanoparticlepolyacrylic acid hydrogel by in situ reduction and polymerization approach. Materials Letters 2014, 116, (0), 135-138.

6. Dispenza, C.; Presti, C. L.; Belfiore, C.; Spadaro, G.; Piazza, S., Electrically conductive hydrogel composites made of polyaniline nanoparticles and poly (N-vinyl-2-pyrrolidone). Polymer 2006, 47, (4), 961-971.

7. Naficy, S.; Razal, J. M.; Spinks, G. M.; Wallace, G. G.; Whitten, P. G., Electrically conductive, tough hydrogels with $\mathrm{pH}$ sensitivity. Chemistry of Materials 2012, 24, (17), 3425-3433.

8. Zhao, Y.; Liu, B.; Pan, L.; Yu, G., 3D nanostructured conductive polymer hydrogels for highperformance electrochemical devices. Energy \& Environmental Science 2013, 6, (10), 28562870 .

9. Lamphere, J. C.; Zweigle, M. L., Cross-linked, water-swellable polymer microgels. Patent No. US4546014 A, 1985

10. Chen, P.; Zhang, W. A.; Luo, W.; Fang, Y. e., Synthesis of superabsorbent polymers by irradiation and their applications in agriculture. Journal of applied polymer science 2004, 93, (4), 1748-1755.

11. Hoffman, A. S., Hydrogels for biomedical applications. Advanced drug delivery reviews 2002, $54,(1), 3-12$.

12. Lira, L. M.; Córdoba de Torresi, S. I., Conducting polymer-hydrogel composites for electrochemical release devices: Synthesis and characterization of semi-interpenetrating polyaniline-polyacrylamide networks. Electrochemistry communications 2005, 7, (7), 717-723.

13. Tang, Q.; Wu, J.; Sun, H.; Fan, S.; Hu, D.; Lin, J., Superabsorbent conducting hydrogel from poly (acrylamide-aniline) with thermo-sensitivity and release properties. Carbohydrate polymers 2008, 73, (3), 473-481.

14. Tang, Q.; Lin, J.; Wu, J.; Zhang, C.; Hao, S., Two-steps synthesis of a poly(acrylate-aniline) conducting hydrogel with an interpenetrated networks structure. Carbohydrate polymers 2007, $67,(3), 332-336$. 